\title{
Correlação entre cardiopatias hipertensivas e aterosclerose na artéria aorta
}

\author{
Pedro Paulo de Mattos Moreno ${ }^{1}$, Mara Lúcia Fonseca Ferraz ${ }^{2}$, Maria Helena Soares ${ }^{3}$, \\ Vicente de Paula Antunes Teixeira ${ }^{4}$, Fernanda Rodrigues Helmo ${ }^{5}$, Rosana Rosa Miranda Corrêa ${ }^{6}$
}

\footnotetext{
${ }^{1}$ Enfermeiro. Residente em Enfermagem Cardiovascular do Instituto Dante Pazzanese. São Paulo, SP, Brasil. E-mail: pedropaulommoreno@hotmail.com.

${ }^{2}$ Bióloga, Doutora em Patologia. Professora do Programa de Pós-Graduação em Ciências da Saúde Geral da Universidade Federal do Triângulo Mineiro. Uberaba, MG, Brasil. E-mail: mara@patge.uftm.edu.br.

${ }^{3}$ Bióloga, Mestre Patologia Básica e Experimental. Técnica em anatomia e necropsia da Universidade Federal do Triângulo Mineiro. Uberaba, MG, Brasil. Email:mhmais@hotmail.com.

${ }^{4}$ Médico, Doutor em Patologia. Professor Titular da Universidade Federal do Triângulo Mineiro. Uberaba, MG, Brasil. Email: vicente@patge.uftm.edu.br.

${ }^{5}$ Enfermeira, Mestre em Patologia. Discente do Programa de Pós-Graduação em Ciências da Saúde Geral, nível Doutorado, da Universidade Federal do Triângulo Mineiro. Uberaba, MG, Brasil. Email: fernandahelmo@gmail.com.

${ }^{6}$ Enfermeira, Doutora em Patologia. Professora Adjunta da Universidade Federal do Triângulo Mineiro. Uberaba, MG, Brasil. E-mail:

rosana.correa@uftm.edu.br.
}

Recebido: 09/04/2016.

Aceito: 22/09/2017.

Publicado: 31/12/2017.

Como citar esse artigo: Moreno PPM, Ferraz MLF, Soares MH, Teixeira VPA, Helmo FR, Corrêa RRM. Correlação entre cardiopatias hipertensivas e aterosclerose na artéria aorta. Rev. Eletr. Enf. [Internet]. 2017 [acesso em:

____;19:a52. Disponível em: http://doi.org/10.5216/ree.v19.40655.

\section{RESUMO}

A hipertensão arterial sistêmica é uma doença multifatorial, comportando-se como fator desencadeador das doenças cardiovasculares e da aterosclerose. O objetivo foi descrever a associação de idade, gênero, cor da pele e o grau de aterosclerose na artéria aorta na cardiopatia hipertensiva em indivíduos autopsiados. Foram avaliados 34 seguimentos de artérias aortas abdominais de indivíduos autopsiados com cardiopatia hipertensiva (20) e sem cardiopatia hipertensiva (14). A idade, gênero, cor da pele e causa de morte foram coletadas do laudo de autopsia, a quantificação da intensidade de aterosclerose e o grau de aterosclerose através de escala padronizada. Neste estudo, a causa de morte e a intensidade da aterosclerose apresentaram associação significativa com a cardiopatia hipertensiva; houve prevalência de placas de ateroma de maior extensão entre os indivíduos com esta entidade. Desse modo, a aterosclerose deve ser investigada em indivíduos com cardiopatia hipertensiva visando prevenir graves repercussões, que podem culminar com o óbito.

Descritores: Aterosclerose. Cardiopatias. Hipertensão Arterial.

\section{INTRODUÇÃO}

A hipertensão arterial sistêmica (HAS) é caracterizada por níveis elevados e sustentados das pressões sistólica e/ou diastólica. A pressão arterial igual a 120/80 mmHg é considerada ótima para adultos. Esta entidade é diagnosticada quando a pressão arterial sistólica apresentase maior ou igual a $140 \mathrm{mmHg}$ e/ou a diastólica maior ou igual a 90 $\mathrm{mmHg}$, em indivíduos acima de 18 anos de idade ${ }^{(1)}$.

Devido às altas taxas de prevalência e a dificuldade de controle, a HAS é considerada um importante 
problema de saúde pública no Brasil e no mundo. Consequentemente, observa-se aumento nos índices de mortalidade por doenças relacionadas à elevação da pressão arterial nos últimos 12 anos, como acidente vascular encefálico, doença isquêmica do coração e aterosclerose em países de baixo e médio desenvolvimento econômico. No Brasil, as doenças cardiovasculares destacam-se entre as principais causas de morte no país, alcançando registros de até 308.466 óbitos/ano decorrentes de doenças do aparelho circulatório ${ }^{(1-2)}$.

A HAS é uma doença multifatorial, sendo seus principais fatores de risco a idade, gênero, etnia, hereditariedade, obesidade, ingestão exacerbada de sal, uso crônico de bebidas alcoólicas, tabagismo, sedentarismo e baixo nível socioeconômico(1). E apesar da alta complexidade, pode ser prevenida e controlada por meio da mudança de hábitos alimentares e estilo de vida ${ }^{(1,3)}$.

Entre as doenças que a HAS pode estar associada, destacam-se as doenças cardiovasculares e as arteriopatias causadas por obstrução de vasos, como a doença isquêmica do coração e a aterosclerose, esta associação resulta em aumento da prevalência da DCV e suas complicações ${ }^{(3)}$. Na progressão da HAS observam-se lesões dos miocardiócitos e hipertrofia do ventrículo esquerdo, caracterizando a cardiopatia hipertensiva. Consequentemente, o músculo cardíaco não é capaz de manter o adequado fluxo sanguíneo, há sobrecarga, hipertrofia das estruturas do miocárdio, neoformação de colágeno e apoptoses cíclicas, as quais desencadeiam desde arritmias graves a paradas cardiorrespiratórias ${ }^{(4)}$.

A aterosclerose é uma doença multifatorial crônica, decorrente da resposta inflamatória à agressão endotelial, acometendo principalmente a camada íntima de artérias de médio e grande calibre. A etiopatogênese desta entidade é caracterizada pelo acúmulo de colesterol e seus ésteres na camada íntima das artérias decorrentes da lesão endotelial prévia ${ }^{(5)}$. 0 acúmulo de lipídio oxidado intersticial favorece a ativação do endotélio vascular e, consequentemente, o recrutamento e ativação de monócitos da circulação. Estas células são responsáveis pela fagocitose dos lipídeos e síntese de citocinas inflamatórias, como a interleucinas-1, que contribuem para a progressão da resposta inflamatória, assim como, recrutamento e ativação de células musculares lisas da camada média para a neoformação de fibras colágenas. É possível observar de forma microscópica o acúmulo de lipídeos tanto no interior de macrófagos e células musculares lisas, denominadas de células espumosas, quanto no interstício vascular, além de áreas de calcificação distrófica. Já as alterações macroscópicas, são caracterizadas pela presença de placas de ateroma de extensão variada, que fazem saliência com a sua luz do vaso. Além disso, algumas placas podem apresentarse fibrosadas ou mesmo calcificadas dependendo da intensidade. Nas lesões iniciais, os macrófagos ativados são responsáveis pela secreção de citocinas e de enzimas proteolíticas e, consequentemente, pela progressão da placa aterosclerótica ${ }^{(6)}$.

Assim, quantificar a espessura da placa de ateroma constitui importante instrumento para a estratificação do risco cardiovascular para o infarto agudo do miocárdio e acidente vascular encefálico, por exemplo, entre os indivíduos acometidos pela cardiopatia hipertensiva ${ }^{(7)}$. Além disso, pesquisa aponta o aumento do risco para doenças cardiovasculares e aterosclerose no período de dez anos devido às 
complicações advindas do envelhecimento associado à HAS nessa população ${ }^{(8)}$. E a literatura mostra-se escassa de estudos que descrevam as características macroscópicas da aterosclerose concomitante a cardiopatia hipertensiva em indivíduos autopsiados ${ }^{(9)}$.

Diante disso, o objetivo deste estudo foi descrever a associação de idade, gênero, cor da pele e o grau de aterosclerose na artéria aorta com a ocorrência de cardiopatia hipertensiva entre os indivíduos autopsiados no Hospital de Clínicas da Universidade Federal do Triângulo Mineiro (HC-UFTM).

\section{MATERIAL E MÉTODOS}

Estudo descritivo e exploratório, no qual foram avaliados retrospectivamente 797 laudos de autopsia entre 1984 a 2014 no HC-UFTM.

Inicialmente foram excluídos 567 casos devido à idade menor que 18 anos, laudos de autopsia incompletos, causa de morte por outro tipo de cardiopatia (ex: miocardiopatia dilatada, cardiopatia reumática, cardiopatia valvular) e ausência de aorta no banco de órgãos.Logo, foram obtidos 230 casos. Destes, foram selecionados 20 segmentos de aorta abdominal de casos com cardiopatia hipertensiva, e 14 segmentos de aorta abdominal de casos sem cardiopatia hipertensiva e outros diagnósticos que interferissem na pressão arterial, como doenças autoimunes e nefropatias. Foram excluídos ainda os casos com aortas danificadas e, portanto, que prejudicavam avaliação macroscópica da aterosclerose, totalizando a exclusão de 196 casos. Assim, foram avaliados 34 seguimentos de artérias aortas abdominais de indivíduos autopsiados com cardiopatia hipertensiva e sem cardiopatia hipertensiva (Figura 1).

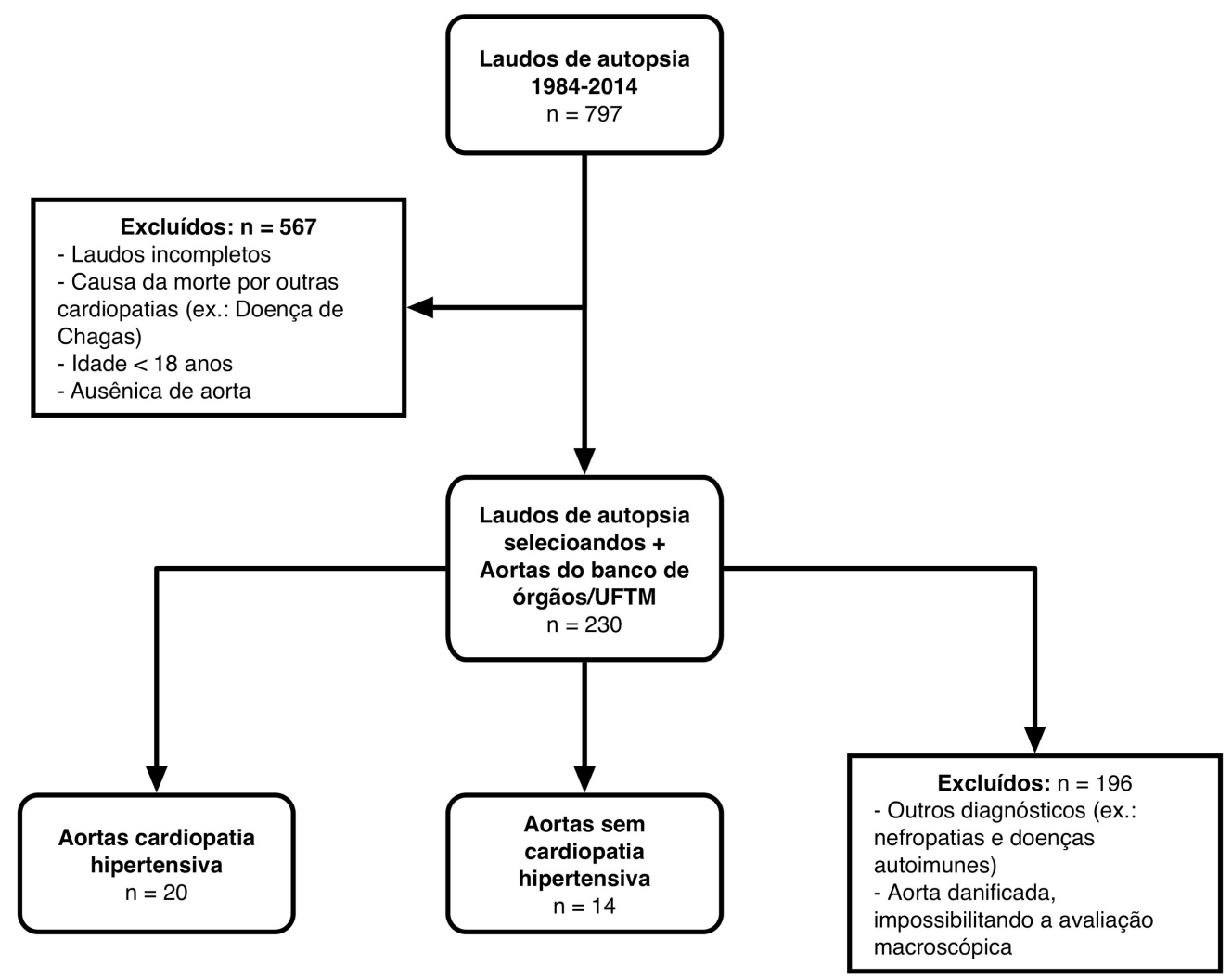

Figura 1: Triagem dos laudos de autopsia e artérias aortas com cardiopatia hipertensiva e sem cardiopatia hipertensiva no período de 1984-2014. 
O delineamento dos grupos foi estabelecido segundo dados da literatura ${ }^{(2)} \mathrm{em}$ :

- Cardiopatia Hipertensiva (CH): indivíduos que desenvolveram aterosclerose em grandes vasos, com diagnóstico de HAS e lesão cardíaca decorrente da HAS; entendendo que as cardiopatias hipertensivas são provenientes de um quadro prolongado de HAS, seguido de lesões dos miocardiócitos e posterior hipertrofia do ventrículo esquerdo.

- Sem Cardiopatia Hipertensiva (SCH): indivíduos sem histórico de HAS, mas que, por outras comorbidades as quais não serão explorados no presente estudo (Diabetes Mellitus ou dislipidemias, por exemplo), também haviam desenvolvido quadro de aterosclerose em grandes vasos.

No laudo de autopsia foram coletadas informações referentes a idade (anos), gênero (masculino/feminino), cor da pele (branco/não branco) e a causa de morte (classificada segundo as entidades mais prevalentes: cardiopatia, infecção, inflamação e neoplasia).

Para a quantificação da aterosclerose cada segmento de artéria aorta abdominal foi avaliado por três observadores. Cada observador analisou o acometimento dos seguimentos da artéria aorta (presença de estrias lipídicas, quantidade de placas lipídicas, extensão da placa, presença de colágeno, hemorragias ou calcificações e integridade de placa aterosclerótica) e registrou de forma subjetiva um ponto em uma escala não milimetrada de $0,0 \mathrm{~cm}$ a $12,0 \mathrm{~cm}$ de comprimento. Em seguida, com um auxílio de uma régua milimetrada, o observador realizou a mensuração da distância de $0,0 \mathrm{~cm}$ ao ponto marcado na escala. Após a quantificação da intensidade de aterosclerose os três observadores consensualmente padronizaram pela média o grau da aterosclerose de acordo com a intensidade em:

1. discreta: de 0,1 a $4,0 \mathrm{~cm}$;

2. moderada: 4,1 a $7,0 \mathrm{~cm}$;

3. acentuada: 7,1 a $12,0 \mathrm{~cm}(9)$.

Uma planilha eletrônica no Microsoft Excel ${ }^{\circledR}$ com todas os dados coletados foi elaborada e as análises estatísticas realizadas por meio do software GraphPad Prism ${ }^{\circledR}$ versão 5.0. Em seguida, as variáveis quantitativas foram testadas para verificar se apresentavam distribuição normal, através do teste de Kolmogorov-Smirnov. As variáveis qualitativas gênero e cor foram analisadas por meio do teste exato de Fisher, enquanto que as várias causas de morte e grau da aterosclerose pelo teste do Chi-quadrado ( $\chi 2$ ); todos resultados foram expressos em números absolutos e porcentagens (\%). Já a variável quantitativa idade (anos) foi expressa em média \pm desvio padrão $(X \pm D P)$ e a comparação das médias analisada através do testet-Student; a intensidade da aterosclerose $(\mathrm{cm})$ foi expressa em mediana e valores mínimos e máximos (Med - Min-Max) e analisada através do teste de Mann-Whitney (T). Foram consideradas estatisticamente significativas as diferenças menores que $5 \%(p<0,05)$.

Esta pesquisa foi aprovada pelo Comitê de Ética em Pesquisa da UFTM (CAAE: 48022015.0.0000.5154).

\section{RESULTADOS}

No presente estudo foram avaliadas 34 amostras de artérias aorta de adultos autopsiados entre 1984 
a 2014. Destes, 59\% (20) apresentavam cardiopatia hipertensiva e média de $61,45 \pm 12,14$ anos, enquanto $41 \%$ (14) não apresentavam e tinham média de 60,50 $\pm 14,85$ anos.

No grupo $\mathrm{CH}$ 15\% (3) eram do gênero feminino e 85\% (17) do masculino; $40 \%$ (8) eram não brancos e $60 \%$ (12) brancos. Enquanto que no grupo SCH 7\% (1) pertenciam ao gênero feminino e 93\% (13) ao masculino; 36\% (5) da amostra era não brancos e 64\% (9) brancos.

A média da idade $(t=0,2049 ; p=0,838)$, o gênero $(p=0,627)$ e a cor $(p=1,000)$ não apresentaram associação significativa entre os grupos avaliados.

A causa de morte apresentou associação significativa em relação à presença de cardiopatia hipertensiva ( $\mathrm{X} 2=8,711 ; p=0,033$ ). No grupo $\mathrm{CH}, 65,0 \%$ (13) dos óbitos foram por cardiopatias e $20,0 \%$ (4) por infecções; enquanto que no grupo SCH esta proporção foi de 35,7\% (5) e 7,1\% (1) respectivamente. Não houve nenhum óbito por neoplasias no primeiro grupo, esta entidade acometeu $28,6 \%$ (4) entre aqueles sem cardiopatia hipertensiva (Figura 2).

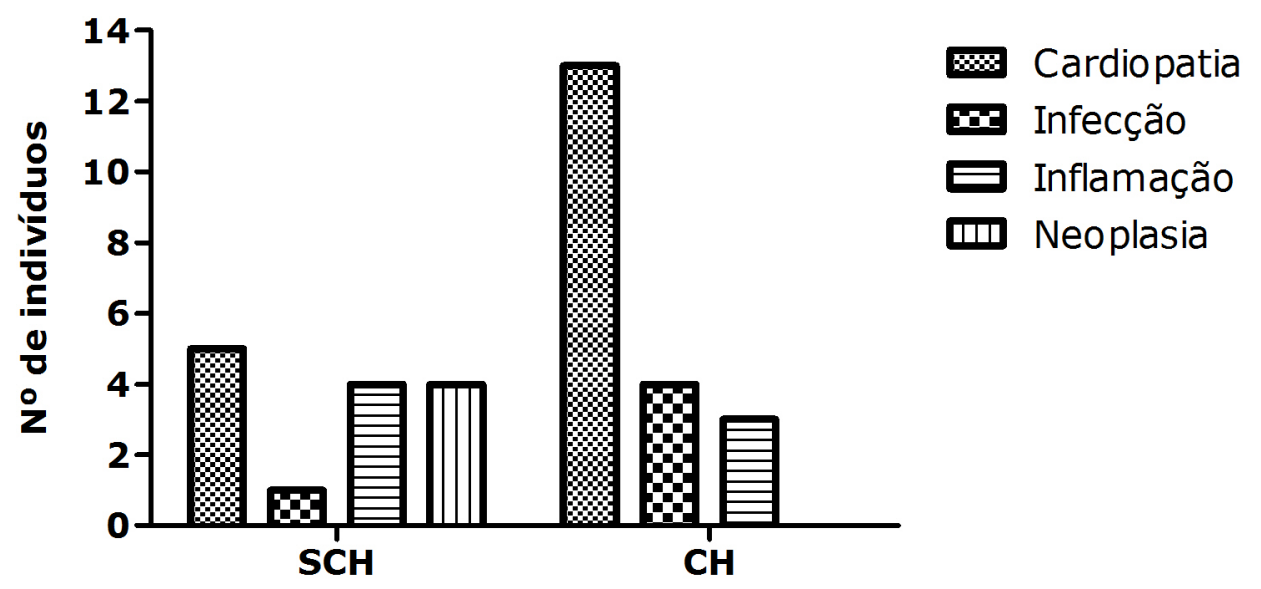

Figura 2: Causa de morte segundo a presença de cardiopatia hipertensiva em autopsias realizadas no Hospital de Clínicas da Universidade Federal do Triângulo Mineiro no período de 1984-2014.

Do mesmo modo, também foi observada associação significativa entre a intensidade da aterosclerose e a presença de cardiopatia hipertensiva $\left(X^{2}=9,166 ; p=0,010\right)$. Visto que no grupo $\mathrm{CH}, 75 \%$ (15) foi classificada como acentuada; enquanto que no grupo SCH, esta proporção foi de $29 \%$ (4) (Tabela 1).

Tabela 1: Intensidade da aterosclerose segundo a presença de cardiopatia hipertensiva em autopsias realizadas no Hospital de Clínicas da Universidade Federal do Triângulo Mineiro de Uberaba, MG, Brasil, no período de 1984-2014 ( $p=0,010)$.

\begin{tabular}{ccc}
\hline Aterosclerose & SCH - \% (n) & CH - \% (n) \\
\hline Discreta & $42(6)$ & $5(1)$ \\
Moderada & $29(4)$ & $20(4)$ \\
Acentuada & $29(4)$ & $\mathbf{7 5 ( 1 5 )}$ \\
\hline Total & $\mathbf{1 0 0 ( 1 4 )}$ & $\mathbf{1 0 0 ( 2 0 )}$ \\
\hline
\end{tabular}

SCH: sem cardiopatia hipertensiva. $\mathrm{CH}$ : cardiopatia hipertensiva.

As placas de ateroma de maior extensão tiveram maior frequência entre os indivíduos com cardiopatia hipertensiva ( $T=63,00 ; p=0,007$ ) (Figura 3). 


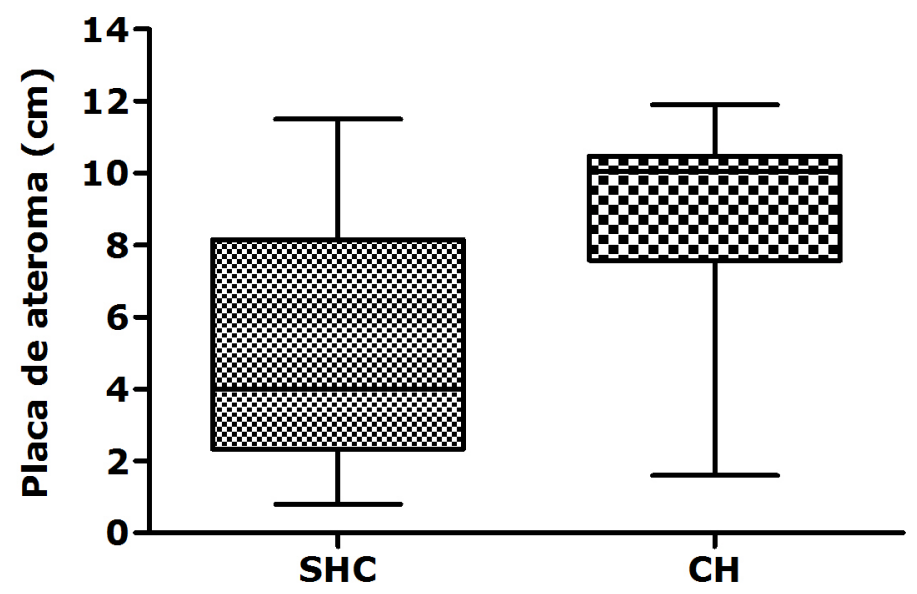

Figura 3: Extensão da placa de ateroma segundo a presença de cardiopatia hipertensiva em autopsias realizadas no Hospital de Clínicas da Universidade Federal do Triângulo Mineiro no período de 1984-2014.

\section{DISCUSSÃO}

Nesta pesquisa, a idade não apresentou influência entre os indivíduos com cardiopatia hipertensiva. Entretanto, dados da literatura apontam a prevalência da HAS e insuficiência cardíaca, por exemplo, em $60 \%^{(1)}$ e $70 \%{ }^{(10)}$ dos indivíduos na faixa etária acima de 60 anos. Além disso, pesquisas visando avaliar a epidemiologia e o risco cardiovascular para insuficiência cardíaca e HAS, apontam média de idade entre 61 e $63,2^{(8)}$ anos, respectivamente, demonstrando, portanto, que os achados desse estudo estão de acordo com a literatura.

Em relação ao gênero e cor da pele, ambos também não apresentaram influência no grupo com cardiopatia hipertensiva neste estudo. Todavia, ambos os fatores mostram-se relevantes na incidência desta entidade, uma vez que pesquisas ressaltam a prevalência de hospitalizações no Brasil por cardiopatia hipertensiva ${ }^{(11)}$ e de hipertensos ${ }^{(12)}$ entre os homens. Em contrapartida, a partir dos 70 anos observa-se maior incidência da hipertensão, acompanhada ou não da hipertrofia do ventrículo esquerdo, entre as mulheres ${ }^{(4)}$. A literatura também enfatiza que a etnia não branca é mais propensa a desenvolver HAS, mas que em países como o Brasil onde há intensa miscigenação ${ }^{(4,11)}$, essa influência pode não ser perceptível entre os indivíduos com $\mathrm{CH}$.

As cardiopatias e as infecções foram as principais causas de morte no grupo com cardiopatia hipertensiva, havendo associação significativa entre as variáveis. As doenças cardiovasculares são responsáveis por $20 \%$ dos óbitos em indivíduos com mais de 30 anos no Brasil, seguido por neoplasias, doenças respiratórias e causas externas ${ }^{(2)}$. Segundo pesquisas que acompanharam hipertensos por um período de até 15 anos, entre $45 \%(13)$ e $60 \%{ }^{(14)}$ dos óbitos foram associados às doenças cardiovasculares, como a doença da artéria coronária ${ }^{(13,15)}$ e insuficiência cardíaca ${ }^{(15)}$. A literatura também ressalta fatores como a obesidade, diabetes mellitus, dislipidemia ${ }^{(2,15)}$, doenças cerebrovasculares e insuficiência renal ${ }^{(13)}$, por exemplo, favorecem o aumento do risco de morte nestes indivíduos.

Ressalta-se ainda que as infecções, como a pneumonia e a sepse, são responsáveis por vários óbitos 
na cardiopatia hipertensiva. Estudos apontam que entre idosos hipertensos e com insuficiência cardíaca prévia, a proporção de óbitos por infecções pode alcançar $37 \%^{(13,16)}$. Recente revisão de literatura ${ }^{(17)}$ salienta ainda que a presença da doença cardíaca crônica favorece o aumento em até 3,3 vezes da pneumonia comunitária, e até 9,9 vezes da doença pneumocócica invasiva de acordo com idade e outros fatores associados como diabetes mellitus, tabagismo, bronquite crônica e asma, confirmando os resultados encontrados neste presente estudo.

Quando investigada a presença de aterosclerose e de cardiopatia hipertensiva, o presente estudo encontrou forte associação, uma vez que $75 \%$ dos indivíduos apresentavam acentuada aterosclerose na artéria aorta. Estudo que avaliou as características morfológicas da aterosclerose em indivíduos autopsiados relata significante correlação entre o grau de aterosclerose e a presença de doença cardiovascular ${ }^{(9)}$.

A dislipidemia favorece a severidade da HAS, uma vez que há associação significativa entre o aumento dos níveis séricos de triglicerídeos, lipoproteína de baixa densidade, lipoproteína de muito baixa densidade e apolipoproteína B100, e aumento da pressão arterial e risco de cardiopatias em indivíduos hipertensos ${ }^{(18)}$. O depósito contínuo de colesterol na camada íntima desencadeia a ativação endotelial, o recrutamento de monócitos e células musculares lisas, a síntese de mediadores inflamatórios e a neoformação de colágeno, por exemplo. Consequentemente, a progressão da aterosclerose está relacionada ao prejuízo da função endotelial e à redução da elasticidade decorrentes do comprometimento da organização, função e vias de sinalização entre os componentes da artéria aorta ${ }^{(19)}$.

Indivíduos com HAS apresentam maior espessura da artéria aorta decorrente da aterosclerose ${ }^{(7,19)} \mathrm{e}$, consequentemente, menor distensibilidade do vaso, quando comparado aos não hipertensos; ressaltando que o aumento da pressão arterial favorece não só os depósitos de gordura, mas também a progressiva rigidez ${ }^{(5,7)}$ e disfunção endotelial da aorta ${ }^{(5)}$ na cardiopatia hipertensiva.

A gravidade da aterosclerose apresenta forte correlação ao aumento do risco de doenças cardiovasculares e não cardiovasculares ${ }^{(19)}$. Estudo aponta que a aterosclerose complicada (placas de ateroma severas, a presença de rupturas, ulcerações e trombos), associada à HAS, diabetes mellitus, dislipidemia e tabagismo, por exemplo, favorece a incidência da doença da artéria coronária ${ }^{(6)}$. A espessura da placa entre 2,1 e 3,$0 ; 3,1$ e 4,0 e 4,1 a 5,0 e $>5,0$ mm, representa um risco de $57 \% ; 60 \%$; $81 \%$ e $86 \%$, respectivamente, para a ocorrência desta entidade ${ }^{(20)}$. De modo semelhante, indivíduos com aterosclerose, hipertensos, índice tornozelo-braquial $\leq 0,9$ e idade maior que 70 anos, apresentam risco aumentado para doença arterial periférica ${ }^{(21)}$.

$\mathrm{Na}$ ausência da cardiopatia hipertensiva, em contrapartida, as lesões ateroscleróticas apresentam-se mais discretas. A literatura ressalta que o envelhecimento e a presença de outras comorbidades, são importantes fatores na progressão da aterosclerose em indivíduos que não apresentam HAS. A intensidade da aterosclerose alcança $53 \%$ destes indivíduos de forma discreta e $19 \%$ de forma moderada. Entre os idosos, principlamente, nota-se uma prevalência da aterosclerose cerca de sete vezes maior em relação aos adultos jovens $^{(22)}$. 
Observou-se a prevalência das placas de ateroma de maior extensão entre os indivíduos com cardiopatia hipertensiva, em relação aos sem cardiopatia hipertensiva. Cerca de $60 \%$ dos indivíduos com doença da artéria coronária ${ }^{(23)}$ ou hipertrofia do ventrículo esquerdo associado à HAS $^{(23-24)}$, diabetes mellitus $^{(23)}$ e dislipidemia ${ }^{(25)}$, por exemplo, apresentam maior incidência de placas complexas na aorta descendente (segmentos torácico e abdominal), extensão e volume até três vezes maiores entre aqueles com cardiopatia hipertensiva ${ }^{(23-24)}$, quando comparados aos normotensos.

Ressalta-se que a literatura aponta média de espessura mínima para a placa de ateroma a partir de $3 \mathrm{~mm}^{(20,25)}$, e máxima $\geq 7 \mathrm{~mm}^{(25)}$ em relação a espessura íntima-média da artéria aorta. Além disso, estima-se que em aproximadamente $35 \%$ e $42 \%$ dos indivíduos com aterosclerose haja a presença significativa de ulcerações e calcificações ${ }^{(25)}$, respectivamente.

Deste modo, percebe-se que a extensão e complexidade da aterosclerose são dependentes do tempo de HAS, idade avançada, aumento da pressão arterial e intenso fluxo sanguíneo, o qual é responsável pela lesão endotelial do vaso.

\section{CONCLUSÃO}

Neste estudo, a idade, o gênero e cor não apresentaram associação entre a presença de aterosclerose e cardiopatia hipertensiva. Entretanto, foi observado que a maioria dos indivíduos com cardiopatia hipertensiva apresentou aterosclerose acentuada e placas de ateroma de maior extensão, sendo as cardiopatias e as infecções as principais causas de morte neste grupo.

A aterosclerose é uma doença complexa, podendo estar associada a diferentes entidades. A dislipidemia, por exemplo, aumenta a gravidade da aterosclerose, da HAS e o risco de cardiopatias em hipertensos. Esses indivíduos apresentam maior espessura da artéria aorta, placas de ateroma extensas, ulceradas e calcificadas e predisposição ao maior risco de morte.

Deste modo, a aterosclerose deve ser considerada nas avaliações de indivíduos com cardiopatia hipertensiva visando prevenir graves repercussões, que podem culminar com o óbito.

\section{Financiamento}

O estudo que originou esse trabalho contou com apoio financeiro das agências:

- Conselho Nacional de Desenvolvimento Cientifico e Tecnológico (CNPq) [número do processo: $470029 / 20110]$

- Coordenação de Aperfeiçoamento de Pessoal de Nível Superior (CAPES) [número do processo: PNPD02604/094];

- Fundação de Amparo a Pesquisa do Estado de Minas Gerais (FAPEMIG) [número do processo: CDSAPQ-02135-14];

- Fundação de Ensino e Pesquisa de Uberaba (FUNEPU) [número do processo: CDS-922/2009]. 


\section{REFERÊNCIAS}

1. VI Diretrizes Brasileiras de Hipertensão. Arq Bras Cardiol [Internet]. 2010 [acesso em: 31 dez. 2017];95(1 Supl 1):151. Disponível em: http://doi.org/10.1590/S0066-782X2010001700001.

2. Mansur AP, Favarato D. Mortality due to cardiovascular diseases in Brazil and in the metropolitan region of São Paulo: a 2011 update. Arq Bras Cardiol [Internet]. 2012 [acesso em: 31 dez. 2017];99(2):755-61. Disponível em: http://doi.org/10.1590/S0066-782X2012005000061.

3. Moraes SA, Freitas ICM. Ischemic heart disease and correlates in adults from Ribeirão Preto, Brazil. Rev Saude Publica [Internet]. 2012 [acesso em: 31 dez. 2017];46(4):591-601. Disponível em: http://doi.org/10.1590/S003489102012005000056.

4. Ribeiro SM, Morceli J, Gonçalves RS, Franco RJ, Habermann F, Meira DA, Matsubara BB. Accuracy of chest radiography plus electrocardiogram in diagnosis of hypertrophy in hypertension. Arq Bras Cardiol [Internet]. 2012 [acesso em: 31 dez. 2017];99(3):825-33. Disponível em: http://doi.org/10.1590/S0066-782X2012005000073.

5. Kopeć G, Podolec P, Podolec J, Rubiś P, Zmudka K, Tracz W. Atherosclerosis progression affects the relationship between endothelial function and aortic stiffness. Atherosclerosis [Internet]. 2009 [acesso em: 31 dez.

2017];204(1):250-4. Disponível em: http://doi.org/10.1016/j.atherosclerosis.2008.09.003.

6. Aono J, Ikeda S, Katsumata Y, Higashi H, Ohshima K, Ishibashi K, et al. Correlation between plaque vulnerability of aorta and coronary artery: an evaluation of plaque activity by direct visualization with angioscopy. Int J Cardiovasc Imaging [Internet]. 2015 [acesso em: 31 dez. 2017];31(6):1107-14. Disponível em: http://doi.org/10.1007/s10554-0150669-z.

7. Liu C-Y, Chen D, Bluemke DA, Wu CO, Teixido-Tura G, Chugh A, et al. Evolution of Aortic Wall Thickness and Stiffness With Atherosclerosis: Long-Term Follow Up From the Multi-Ethnic Study of Atherosclerosis. Hypertension [Internet]. 2015 [acesso em: 31 dez. 2017];65(5):1015-9. Disponível em: http://doi.org/10.1161/HYPERTENSIONAHA.114.05080. 8. Cesarino EJ, Vituzzo AL, Sampaio JM, Ferreira DA, Pires HA, Souza L. Assessment of cardiovascular risk of patients with arterial hypertension of a public health unit. Einstein (São Paulo) [Internet]. 2012 [acesso em: 31 dez.

2017];10(1):33-8. Disponível em: http://doi.org/10.1590/S1679-45082012000100008.

9. Ferraz ML, Nascimento DM, Rorato JP, Espindula AP, Oliveira LF, Ramalho LS, et al.Correlation of lifetime progress of atherosclerosis and morphologic markers of severity in humans: new tools for a more sensitive evaluation. Clinics [Internet]. 2012 [acesso em: 31 dez. 2017];67(9):1071-5. Disponível em: http://doi.org/10.6061/clinics/2012(09)15.10. Bocchi EA. Heart failure in South America. Curr Cardiol Rev [Internet]. 2013 [acesso em: 31 dez. 2017];9(2):147-56. Disponível em: http://doi.org/10.2174/1573403X11309020007.

11. Hartmann M, Dias-da-Costa JS, Olinto MTA, Pattussi MP, Tramontini Â. Prevalência de hipertensão arterial sistêmica e fatores associados: um estudo de base populacional em mulheres no Sul do Brasil. Cad Saude Publica [Internet]. 2007 [acesso em: 31 dez. 2017];23(8):1857-66. Disponível em: http://doi.org/10.1590/S0102311X2007000800012.

12. Gus I, Harzheim E, Zaslavsky C, Medina C, Gus M. Prevalência, reconhecimento e controle da hipertensão arterial sistêmica no estado do Rio Grande do Sul. Arq Bras Cardiol [Internet]. 2004 [acesso em: 31 dez. 2017];83(5):424-8. Disponível em: http://doi.org/10.1590/S0066-782X2004001700009.

13. Cui H, Hu Y, Hong C, Hu G, Fan L. A 15 years study of the causes of death among elderly hypertensive patients in a hospital-based sample of China. Arch Gerontol Geriatr [Internet]. 2012 [acesso em: 31 dez. 2017];55(3):709-12. Disponível em: https://doi.org/10.1016/j.archger.2012.07.008.

14. Okin PM, Kjeldsen SE, Julius S, Hille DA, Dahlöf B, Edelman JM, et al. All-cause and cardiovascular mortality in relation to changing heart rate during treatment of hypertensive patients with electrocardiographic left ventricular hypertrophy. Eur Heart J [Internet]. 2010 [acesso em: 31 dez. 2017];31(18):2271-9. Disponível em:

http://doi.org/10.1093/eurheartj/ehq225.

15. Chen Q, Smith CY, Bailey KR, Wennberg PW, Kullo IJ. Disease Location Is Associated With Survival in Patients With Peripheral Arterial Disease. J Am Heart Assoc [Internet]. 2013 [acesso em: 31 dez. 2017];2(5):e000304-e000304. Disponível em: https://doi.org/10.1161/JAHA.113.000304.

16. Ueda T, Kawakami R, Horii M, Sugawara Y, Matsumoto T, Okada S, et al. Noncardiovascular Death, Especially Infection, Is a Significant Cause of Death in Elderly Patients With Acutely Decompensated Heart Failure. J Card Fail [Internet]. 2014 [acesso em: 31 dez. 2017];20(3):174-80. Disponível em:

http://doi.org/10.1016/j.cardfail.2013.12.007.

Rev. Eletr. Enf. [Internet]. 2017 [acesso em:_____];19:a52. Disponível em: http://doi.org/10.5216/ree.v19.40655. 
17. Torres A, Blasi F, Dartois N, Akova M. Which individuals are at increased risk of pneumococcal disease and why? Impact of COPD, asthma, smoking, diabetes, and/or chronic heart disease on community-acquired pneumonia and invasive pneumococcal disease. Thorax [Internet]. 2015 [acesso em: 31 dez. 2017];70(10):984-9. Disponível em: http://doi.org/10.1136/thoraxjnl-2015-206780.

18. Nayak P, Panda S, Thatoi PK, Rattan R, Mohapatra S, Mishra PK. Evaluation of Lipid Profile and Apolipoproteins in Essential Hypertensive Patients. J Clin Diagn Res [Internet]. 2016 [acesso em: 31 dez. 2017];10(10):BC01-BC04. Disponível em: http://doi.org/10.7860/JCDR/2016/20985.8626.

19. Maroules CD, Rosero E, Ayers C, Peshock RM, Khera A. Abdominal aortic atherosclerosis at MR imaging is associated with cardiovascular events: the Dallas heart study. Radiology [Internet]. 2013 [acesso em: $31 \mathrm{dez}$. 2017];269(1):84-91. Disponível em: http://doi.org/10.1148/radiol.13122707.

20. Couturier G, Voustaniouk A, Weinberger J, Fuster V. Correlation between coronary artery disease and aortic arch plaque thickness measured by non-invasive B-mode ultrasonography. Atherosclerosis [Internet]. 2006 [acesso em: 31 dez. 2017];185(1):159-64. Disponível em: http://doi.org/10.1016/j.atherosclerosis.2005.05.035.

21. Brasileiro AC, Oliveira DC, Victor EG, Oliveira DA, Batista LL. Association between ankle-brachial index and carotid atherosclerotic disease. Arq Bras Cardiol [Internet]. 2013 [acesso em: 31 dez. 2017];100(5):422-8. Disponível em: http://doi.org/10.5935/abc.20130057.

22. Webber BJ, Seguin PG, Burnett DG, Clark LL, Otto JL. Prevalence of and risk factors for autopsy-determined atherosclerosis among US service members, 2001-2011. JAMA [Internet]. 2012 [acesso em: $31 \mathrm{dez}$.

2017];308(24):2577-83. Disponível em: http://doi.org/10.1001/jama.2012.70830.

23. Gu X, He Y, Li Z, Kontos MC, Paulsen WH, Arrowood JA, Vetrovec GW, Nixon JV. Relation between the incidence, location, and extent of thoracic aortic atherosclerosis detected by transesophageal echocardiography and the extent of coronary artery disease by angiography. Am J Cardiol [Internet]. 2011 [acesso em: 31 dez. 2017];107(2):175-8. Disponível em: http://doi.org/10.1016/j.amjcard.2010.09.003.

24. Courand P-Y, Milon H, Bricca G, Khettab F, Lantelme P. Diastolic blood pressure, aortic atheroma, and prognosis in hypertension: New insights into a complex association. Atherosclerosis [Internet]. 2014 [acesso em: 31 dez.

2017];233(1):300-6. Disponível em: http://doi.org/10.1016/j.atherosclerosis.2014.01.004.

25. Mizuma A, Kijima C, lijima K, Goto Y, Honma K, Yasuda T, et al. Relationship between Atherosclerotic Risk Factors and Aortic Plaques in Patients with First-ever Ischaemic Stroke. Hear Lung Circ [Internet]. 2014 [acesso em: 31 dez. 2017];23(10):930-5. Disponível em: http://doi.org/10.1016/j.hlc.2014.02.013. 